\title{
ON PAIRWISE PARACOMPACTNESS
}

\author{
M. GANSTER and I. L. REILLY
}

(Received 30 May 1990)

Communicated by J. H. Rubinstein

\begin{abstract}
This paper answers a recent question concerning the relationship between two notions of paracompactness for bitopological spaces. Romaguera and Marin defined pairwise paracompactness in terms of pair open covers, motivated by a characterization of paracompactness due to Junnila. On the other hand, Raghavan and Reilly defined a bitopological space $(X, \tau, \sigma)$ to be $\delta$-pairwise paracompact if and only if every $\tau$ open ( $\sigma$ open) cover of $X$ admits a $\tau \vee \sigma$ open refinement which is $\tau \vee \sigma$ locally finite. It is shown that pairwise paracompactness implies $\delta$-pairwise paracompactness, and that the converse is false.
\end{abstract}

1991 Mathematics subject classification (Amer. Math. Soc.): 54 D 18, 54 E 55.

Keywords and phrases: bitopological space, pairwise paracompact, pairwise regular.

One of the many results for which Bing will be remembered is the theorem that a topological space is metrizable if and only if it is paracompact and developable [1]. Recently, Romaguera and Marin [6, Theorem 1] have extended this result to the class of bitopological spaces. To do this, they first had to make appropriate definitions of pairwise paracompactness and pairwise developability for the category of bitopological spaces. The definitions relevant to our discussion are as follows, where $\mathbb{N}$ denotes the set of non-negative integers.

DefINITION 1. A pair open cover in a bitopological space $(X, \tau, \sigma)$ is a family of pairs $\left\{\left(G_{\alpha}, H_{\alpha}\right): \alpha \in I\right\}$ such that

(i) for each $\alpha \in I, G_{\alpha}$ is a $\tau$ open set and $H_{\alpha}$ is a $\sigma$ open set,

(ii) $\mathscr{G}=\left\{G_{\alpha}: \alpha \in I\right\}$ and $\mathscr{H}=\left\{H_{\alpha}: \alpha \in I\right\}$ are covers of $X$,

(iii) for each $x \in X$ there is an $\alpha \in I$ such that $x \in G_{\alpha} \cap H_{\alpha}$.

(C) 1992 Australian Mathematical Society $0263-6115 / 92 \$ A 2.00+0.00$ 
Such a pair open cover is denoted by $(\mathscr{G}, \mathscr{H})$.

In [3] Junnila obtained the following characterization of paracompactness. A regular topological space $X$ is paracompact if, and only if, given an open cover $\mathscr{G}$ of $X$, then for each $x \in X$ there is a sequence $\left\{U_{n}(x): n \in \mathbb{N}\right\}$ of neighbourhoods of $x$ such that

(i) $y \in U_{n}(x)$ if and only if $x \in U_{n}(y)$,

(ii) if $x \in X$ there is an $n \in N$ and a $G \in \mathscr{G}$ with $U_{n}^{2}(x) \subset G$, where

$$
U_{n}^{2}(x)=\bigcup\left\{U_{n}(y): y \in U_{n}(x)\right\}
$$

This result of Junnila motivated the next definition of Romaguera and Marin [6, Definition 4]. Recall that for the bitopological space $(X, \tau, \sigma)$ we say that $\tau$ is regular with respect to $\sigma$ if for each point $x$ in $X$ and each $\tau$ closed set $K$ such that $x \notin K$ there is a $\tau$ open set $U$ and a $\sigma$ open set $V$ disjoint from $U$ such that $x \in U$ and $K \subset V$, or equivalently if for each point $x$ in $X$ and $\tau$ open set $G$ containing $x$ there is a $\tau$ open set $H$ such that $x \in H \subset \sigma \mathrm{cl} H \subset G$. Then a bitopological space $(X, \tau, \sigma)$ is called pairwise regular if $\tau$ is regular with respect to $\sigma$ and $\sigma$ is regular with respect to $\tau$.

DEFINITION 2. A pairwise regular bitopological space $(X, \tau, \sigma)$ is pairwise paracompact if given a pair open cover $(\mathscr{G}, \mathscr{H})$ of $(X, \tau, \sigma)$ then for each $x \in X$ there is a sequence $\left\{U_{n}(x): n \in \mathbb{N}\right\}$ of $\tau$ neighbourhoods of $x$ and a sequence $\left\{V_{n}(x): n \in \mathbb{N}\right\}$ of $\sigma$ neighbourhoods of $x$ such that

(i) $y \in U_{n}(x)$ if and only if $x \in V_{n}(y)$,

(ii) if $x \in X$ there is an $n \in N$ and a pair $\left(G_{\alpha}, H_{a}\right) \in(\mathscr{G}, \mathscr{H})$ with $U_{n}^{2}(x) \subset G_{a}$ and $V_{n}^{2}(x) \subset H_{\alpha}$.

In an earlier attempt to provide a useful definition of bitopological paracompactness, Raghavan and Reilly [5, Definition 3.1] introduced several notions, the most general of which was the following.

DEFINITION 3. A bitopological space $(X, \tau, \sigma)$ is $\delta$-pairwise paracompact if every $\tau$ open ( $\sigma$ open) cover of $X$ has a $\tau \vee \sigma$ open refinement which is $\tau \vee \sigma$ locally finite.

This definition allowed a bitopological version of Michael's classical characterization of regular paracompact spaces.

The question of the relationship between these two forms of bitopological paracompactness was raised by Romaguera and Marin $[6, \S 5]$, and it is the purpose of this paper to answer that question.

It is clear that if $(X, \tau, \sigma)$ is a bitopological space such that $(X, \tau \vee \sigma)$ is paracompact, then $(X, \tau, \sigma)$ is $\delta$-pairwise paracompact. The following example, due to Girhiny [2, Proposition 4], shows that the converse is false. 
EXAMPLE 1. The Sorgenfrey plane is not paracompact, but it can be represented as the join of two paracompact topologies. Specifically, let $X$ be the plane, let $\tau$ be the product of the usual topology $\mu$ on the reals $\mathbb{R}$ with the topology on $\mathbb{R}$ with base the half-open intervals of the form $[a, b)$, and let $\sigma$ be the product of the topology on $\mathbb{R}$ with base the half-open intervals of the form $[a, b)$ with the usual topology $\mu$ on $\mathbb{R}$. Then the Sorgenfrey topology $\mathscr{T}$ on $X$ is the joint $\tau \vee \sigma$, and is not paracompact. However, each of the topologies $\tau$ and $\sigma$ is paracompact, because it is the product of a paracompact $T_{3}$ space and a topology which is countable at infinity, Kowalsky [4, page $153,22.8]$. Moreover, $(X, \tau, \sigma)$ is clearly $\delta$-pairwise paracompact.

Our observations above show that for an arbitrary bitopological space $(X, \tau, \sigma)$, the condition that $(X, \tau, \sigma)$ is $\delta$-pairwise paracompact is strictly weaker than the condition that $(X, \tau \vee \sigma)$ is paracompact. According to Definition 2, pairwise paracompactness is defined only for pairwise regular spaces, so that any finite bitopological space which is not pairwise regular is $\delta$-pairwise paracompact but not pairwise paracompact. However, we shall show in Proposition 1 and Example 2 that for pairwise regular spaces pairwise paracompactness is strictly stronger than $\delta$-pairwise paracompactness.

Proposition 1. Let $(X, \tau, \sigma)$ be pairwise regular. If $(X, \tau, \sigma)$ is pairwise paracompact then $(X, \tau \vee \sigma)$ is paracompact.

Proof. First we show that $(X, \tau \vee \sigma)$ is regular. Let $W \in \tau \vee \sigma$ and $x \in W$. So there are sets $U \in \tau$ and $V \in \sigma$ such that $x \in U \cap V \subset W$. By the pairwise regularity of $(X, \tau, \sigma)$, there is a set $U_{1} \in \tau$ such that $x \in U_{1} \subset \sigma \mathrm{cl} U_{1} \subset U$, and a set $V_{1} \in \sigma$ such that $x \in V_{1} \subset \tau \mathrm{cl} V_{1} \subset V$. Here $\mu \mathrm{cl} A$ denotes the closure of $A$ with respect to the topology $\mu$. Then $x \in U_{1} \cap V_{1}$ and $U_{1} \cap V_{1} \in \tau \vee \sigma$, while we have $(\tau \vee \sigma) \operatorname{cl}\left(U_{1} \cap V_{1}\right) \subset \sigma \operatorname{cl}\left(U_{1} \cap\right.$ $\left.V_{1}\right) \subset \sigma \operatorname{cl} U_{1} \subset U$, and $(\tau \vee \sigma) \operatorname{cl}\left(U_{1} \cap V_{1}\right) \subset \tau \operatorname{cl}\left(U_{1} \cap V_{1}\right) \subset \tau \operatorname{cl} V_{1} \subset V$. Hence $(\tau \vee \sigma) \operatorname{cl}\left(U_{1} \cap V_{1}\right) \subset U \cap V \subset W$. Thus $\tau \vee \sigma$ is regular.

Next we use Junnila's theorem to show that $(X, \tau \vee \sigma)$ is paracompact. Let $\mathscr{U}$ be an open cover of $(X, \tau \vee \sigma)$. Without loss of generality, we assume that $\mathscr{U}$ consists of $(\tau \vee \sigma)$ basic open sets, that is $\mathscr{U}=\left\{U_{\alpha} \in \tau \vee \sigma: U_{\alpha}=G_{\alpha} \cap H_{\alpha}\right.$, $\left.G_{\alpha} \in \tau, H_{\alpha} \in \sigma\right\}$. Then $\left\{\left(G_{\sigma}, H_{\sigma}\right)\right\}$ is a pair open cover of $(X, \tau, \sigma)$, which is pairwise paracompact. Let $x \in X$, and $\left\langle U_{n}(x)\right\rangle$ and $\left\langle V_{n}(x)\right\rangle$ be the sequences satisfying Definition 2. Define $W_{n}(x)=U_{n}(x) \cap V_{n}(x)$. Then $W_{n}(x) \in \tau \vee \sigma$. Condition (i) of Definition 2 shows that $y \in W_{n}(x)$ if and only if $x \in W_{n}(y)$. Furthermore, if $x \in X$ by condition (ii) of Definition 2 
there is an $n \in \mathbb{N}$ and a pair $\left(G_{\alpha}, H_{\alpha}\right)$ such that $U_{n}^{2}(x) \subset G_{\alpha}$ and $V_{n}^{2}(x) \subset$ $H_{\alpha}$. Then $W_{n}^{2}(x) \subset U_{\alpha}$. Thus $(X, \tau \vee \sigma)$ is paracompact, by Junnila's theorem.

Proposition 1 and the observations preceding Example 1 together prove the following result.

Proposition 2. Let $(X, \tau, \sigma)$ be pairwise regular. If $(X, \tau, \sigma)$ is pairwise paracompact then it is $\delta$-pairwise paracompact.

We now show that the converse of Proposition 1 is false.

EXAMPLe 2. Let $X$ be the set of real numbers, $\tau$ be the usual topology and $\sigma$ be the discrete topology. First we note that $(X, \tau, \sigma)$ is pairwise regular. Then $\tau \vee \sigma=\sigma$, so $(X, \tau \vee \sigma)$ is paracompact. Now suppose that $(X, \tau, \sigma)$ is pairwise paracompact. Consider the pair open cover $(\mathscr{G}, \mathscr{H})$ of $(X, \tau, \sigma)$, where $\mathscr{G}=\left\{G_{x}: x \in X\right\}, \mathscr{H}=\left\{H_{x}: x \in X\right\}$, and for each $x \in X$ we choose $G_{x}=X$ and $H_{x}=\{x\}$. Suppose $\left\langle U_{n}(x)\right\rangle$ and $\left\langle V_{n}(x)\right\rangle$ are sequences satisfying Definition 2. For $x \in X$, there is an $n \in \mathbb{N}$ such that $x \in V_{n}(x) \subset V_{n}^{2}(x) \subset\{x\}$, so that $V_{n}(x)=\{x\}$. That is, for each $x \in X$, there is an $n_{x} \in \mathbb{N}$ such that $V_{n}(x)=\{x\}$. For each $n \in \mathbb{N}$, let $X_{n}=\left\{x \in X: n_{x}=n\right\}$. Then $X=\bigcup\left\{X_{n}: n \in \mathbb{N}\right\}$. So there is an $m \in \mathbb{N}$ such that $X_{m}$ is uncountable. If $y \in X_{m}$ then $U_{m}(y) \cap X_{m}=\{y\}$. For otherwise, if $x \neq y$ and $x \in U_{m}(y) \cap X_{m}$ then (i) of Definition 2 implies that $y \in V_{m}(x)=\{x\}$, a contradiction. Thus $X_{m}$ is an uncountable discrete subspace of the reals with the usual topology, which is impossible. Hence $(X, \tau, \sigma)$ is not pairwise paracompact.

QUESTION. Do any of the results of Romaguera and Marin [6], especially their Theorem 1, remain true if "pairwise paracompact" is replaced by " $\delta$ pairwise paracompact" or by " $(X, \tau \vee \sigma)$ is paracompact"?

\section{References}

[1] R. H. Bing, 'Metrization of topological spaces', Canad. J. Math. 3 (1951), 175-186.

[2] J. Girhiny, 'Preservation of topological properties under lattice operations and relations', McMaster Mathematical Report 33 (1970).

[3] H. J. K. Junnila, Covering properties and quasi-uniformities of topological spaces, Thesis, Virginia Polytechnic Institute and State University (1978).

[4] H. J. Kowalsky, Topological Spaces (Academic Press, New York, 1965).

[5] T. G. Raghavan and I. L. Reilly, 'A new bitopological paracompactness', J. Austral. Math. Soc. (Series A) 41 (1986), 268-274. 
[6] S. Romaguera and J. Marin, 'On the bitopologial extension of the Bing metrization theorem', J. Austral. Math. Soc. (Series A) 44 (1988), 233-241.

Institut für Mathematik

Technische Universität Graz

Graz

Austria
Department of Mathematics and

Statistics

University of Auckland

Auckland

New Zealand 